\title{
Effect of rosuvastatin on vascular endothelial functions and inflammatory factors of patients with type 2 diabetes mellitus and coronary heart disease
}

\author{
GANG MA and SHUTING BI \\ Department of Cardiac Surgery, Center Hospital of Zibo, Zibo, Shandong 255036, P.R. China
}

Received September 14, 2017; Accepted October 3, 2018

DOI: $10.3892 /$ etm.2018.6923

\begin{abstract}
Effects of rosuvastatin on vascular endothelial functions and inflammatory factors of patients with type 2 diabetes mellitus and coronary heart disease were investigated. Eighty patients with type 2 diabetes mellitus and coronary heart disease, who were admitted and treated in Center hospital of Zibo from January 2016 to January 2017, were selected and divided into observation group $(n=40)$ and control group $(n=40)$ by the random number table; symptomatic and supporting therapy, including use of metformin, captopril, asprin and levocarnitine, was used in control group while rosuvastatin was adopted in observation group in addition to the symptomatic and supporting therapy. Patients in both groups were treated for a treatment cycle, namely, 3 consecutive months. After that, indexes related to blood lipid, diabetes mellitus and vascular endothelial activity, as well as variations in inflammation-associated cytokines, before and after intervention were compared; the correlation of changes in total cholesterol (TC) with those in fasting insulin (FINS), high-sensitivity C-reactive protein (hs-CRP) and endothelin-1 (ET-1), respectively, was analyzed. Among the blood lipid indexes of the patients, the levels of TC, triglyceride (TG) and low-density lipoprotein cholesterol (LDL-C) after intervention were significantly lower than those before intervention $(\mathrm{P}<0.05)$, while the post-intervention level of high-density lipoprotein cholesterol (HDL-C) was higher than that before intervention $(\mathrm{P}<0.05)$. Compared with those before intervention, the level of FINS after intervention was remarkably higher $(\mathrm{P}<0.05)$, while the homeostasis model assessment of insulin resistance (HOMA-IR) level after intervention was significantly lower $(\mathrm{P}<0.05)$. After intervention, the levels of hs-CRP and tumor necrosis factor- $\alpha$ (TNF- $\alpha)$ in the patients were obviously decreased compared with those
\end{abstract}

Correspondence to: Dr Gang Ma, Department of Cardiac Surgery, Center Hospital of Zibo, 54 Gongqingtuan Road, Zhangdian, Zibo, Shandong 255036, P.R. China

E-mail: magangzb@126.com

Key words: rosuvastatin, type 2 diabetes mellitus, patients with coronary heart disease, vascular endothelial functions, inflammatory factors, correlation research before intervention $(\mathrm{P}<0.05)$. Compared with that before intervention, the ET-1 level was decreased $(\mathrm{P}<0.05)$, while the nitric oxide (NO) level was elevated after intervention $(\mathrm{P}<0.05)$. The TC level was negatively correlated with FINS level $(\mathrm{P}<0.05)$ but positively correlated with the levels of hs-CRP $(\mathrm{P}<0.05)$ and ET-1 $(\mathrm{P}<0.05)$. For patients with type 2 diabetes mellitus and coronary heart disease, treatment with rosuvastatin can effectively lower the level of blood lipid and regulate insulin functions; moreover, potent decrease in blood lipid level has great significance in improving the vascular endothelial functions and reducing inflammatory response levels.

\section{Introduction}

Patients with type 2 diabetes mellitus and coronary heart disease usually have abnormal blood lipids, vascular endothelial dysfunctions, insulin resistance and increased inflammatory cells (1). For patients with type 2 diabetes mellitus and coronary heart disease, the comprehensive effect of multiple risk factors can lead to rapid progression of the diseases, such as severe complications of the heart, brain, liver, kidney and other important organs in the early stage (2). Studies have confirmed that increase in blood lipids, injury of vascular endothelial cells, increase in inflammatory responses and insulin resistance are the independent risk factors for the occurrence and development of coronary heart disease and (or) type 2 diabetes mellitus (3). Therefore, adjusting the level of blood lipids effectively has significant value to alleviate the severity, slow the progression and reduce the occurrence of complications of type 2 diabetes mellitus and coronary heart disease (4).

Statins can play a role in regulating the blood lipids through selectively competitive inhibition of 3-hydroxy-3-methylglutaryl coenzyme A (HMG-CoA) reductase (5). As to treatment of coronary heart disease, statins can effectively lower the levels of serum total cholesterol (TC), triglyceride (TG) and other indexes related to blood lipids; moreover, they can increase the level of high-density lipoprotein cholesterol (HDL-C), slow the progression of atherosclerosis, stabilize plaques and reduce the risk of thromboembolism (6). For patients with diabetes mellitus, statins have certain functions of lowering inflammatory response factor level in the body, improving the vascular endothelial functions, exerting anti-oxidation and enhancing insulin sensitivity (7). Rosuvastatin is a kind 
of water-soluble statin that is often used at present; compared with lipid-soluble statins, it has shorter onset time and better efficacy in clinic. Although there have been studies on the treatment of diabetes mellitus, coronary heart disease and hyperlipidemia with rosuvastatin in clinical practice (8), after the treatment with rosuvastatin of type 2 diabetes mellitus combined with coronary heart disease, the blood lipid index and insulin resistance, the correlation between the changes of inflammatory factors and the function of endothelial cells is rarely reported. This research focused on treatment with rosuvastatin on patients with diabetes mellitus and coronary heart disease and investigated its effect on vascular endothelial functions and inflammatory factors in the body.

\section{Materials and methods}

General information. Eighty patients with type 2 diabetes mellitus and coronary heart disease, who were admitted and treated in Center hospital of Zibo (Zibo, China) from January 2016 to January 2017, were selected; for all the enrolled patients, the coronary heart disease was confirmed by coronary angiography, and type 2 diabetes mellitus was confirmed by glucose tolerance test and glucose determination. Before enrollment, the patients were advised, and signed the informed consent; and, this study was approved by the Ethics Committee of Center Hospital of Zibo (no. ZBCH2015-03). Patients who were complicated with systemic infection, malignant tumors, mental diseases, systemic immune system dysfunctions or other endocrine system diseases, who had been treated with glucocorticoid and (or) immunodepressant for 3 months before enrollment, and who signed the informed consent but refused to be enrolled, were excluded. All the patients were divided into observation group $(n=40)$ and control group $(n=40)$ by the random number table. In observation group, there were 26 men and 14 women aged 50-75 years, with an average age of $67.1 \pm 1.2$ years; the duration of diabetes mellitus was 5-40 years, with an average duration of $25.1 \pm 2.1$ years, and the duration of coronary heart disease was 5-30 years, with an average duration of $18.2 \pm 1.0$ years. In control group, there were 25 men and 15 women aged 50-75 years, with an average age of $67.0 \pm 1.3$ years; the duration of diabetes mellitus was

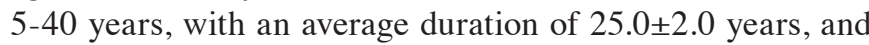
the duration of coronary heart disease was 5-30 years, with an average duration of $18.1 \pm 0.9$ years. The differences in sex, age as well as duration of diabetes mellitus and coronary heart disease between the two groups were not statistically significant $(\mathrm{P}>0.05)$.

Methods. Symptomatic and supporting therapy was used for patients with type 2 diabetes mellitus and coronary heart disease in control group, including oral administration of metformin (0.5 g, b.i.d. National Medicine Permission No. H11021518, Beijing Jingfeng Pharmaceutical Co., Ltd., Beijing, China) for blood glucose control, Captopril (12.5-25.0 mg 3 times a day, National Medicine Permission No. : H44024904, Shantou Jinshi Pharmaceutical General Factory, Shezhen, China) for blood pressure regulation, anticoagulation, Enteric Aspirin Tablets (100 mg daily before bedtime, National Medicine Permission No. H20065051 Shenyang Orge Pharmaceutical Co., Ltd., Liaoning, China) for platelet inhibition, oral
Table I. Comparison of indexes related to blood lipid before and after intervention $(\mathrm{mmol} / \mathrm{l}$, mean $\pm \mathrm{SD})$.

\begin{tabular}{lcccc}
\hline & TC & TG & LDL-C & HDL-C \\
\hline Before intervention & $6.24 \pm 0.02$ & $2.41 \pm 0.02$ & $3.54 \pm 0.02$ & $1.13 \pm 0.01$ \\
After intervention & $5.41 \pm 0.01$ & $1.86 \pm 0.01$ & $2.55 \pm 0.02$ & $1.42 \pm 0.02$ \\
t value & 234.759 & 155.563 & 221.371 & 73.539 \\
P-value & $<0.001$ & $<0.001$ & $<0.001$ & $<0.001$ \\
\hline
\end{tabular}

Table II. Comparison of FINS and HOMA-IR levels before and after intervention (mean $\pm \mathrm{SD}$ ).

\begin{tabular}{lcr}
\hline & FINS (mU/l) & HOMA-IR \\
\hline Before intervention & $4.6 \pm 0.1$ & $1.4 \pm 0.2$ \\
After intervention & $8.5 \pm 0.3$ & $1.0 \pm 0.1$ \\
t value & 78.000 & 11.314 \\
P-value & $<0.001$ & $<0.001$ \\
\hline
\end{tabular}

administration of levocarnitine ( $3 \mathrm{~g}$ per day, three times a day, National Medicine Permission No. H19990372, Northeastern Pharmaceutical Group Shenyang First Pharmaceutical Co., Ltd., Liaoning, China) heart protection; in observation group, rosuvastatin (Simcere Pharmaceutical Group, Nanjing, China; National Medicine Permission No. H20113246) was adopted on the basis of treatment for control group. The drug (10 mg) was orally administered once at bedtime every day for a 3-month treatment cycle. Outpatient follow-up was conducted for all the patients.

Observation indexes. Indexes related to blood lipids [such as TC, TG, low density lipoprotein cholesterol (LDL-C) and HDL-C] (Cholesterol determination kit, cat. no. YZB/Gui 0128-2013; Sichuan Mike Biotechnology Co., Ltd., Chengdu, China), diabetes mellitus [fasting insulin (FINS) and homeostasis model assessment of insulin resistance (HOMA-IR)], inflammation-associated cytokines [including variations in tumor necrosis factor-alpha (TNF- $\alpha$ ) and high-sensitivity C-reactive protein (hs-CRP)] and vascular endothelial activity [changes in endothelin-1 (ET-1) and nitric oxide (NO) levels] before and after intervention were compared; the correlations of changes in TC with those in FINS, hs-CRP and ET-1 were analyzed.

Evaluation indexes. The enrolled patients underwent more than $8 \mathrm{~h}$ of fasting, followed by collection of venous blood from the elbow in the morning, which was taken as the specimen. The main detections for the indexes related to blood lipids included TC $(<5.20 \mathrm{mmol} / \mathrm{l})$, TG $(<1.70 \mathrm{mmol} / \mathrm{l})$, LDL-C $(<3.12 \mathrm{mmol} / \mathrm{l})$ and HDL-C $(>1.04 \mathrm{mmol} / \mathrm{l})$. All those detections were performed using Aeroset automatic biochemistry analyzer (Abbott Pharmaceutical Co. Ltd., Lake Bluff, IL, USA). HOMA-IR = [fasting blood glucose $(\mathrm{mmol} / \mathrm{l}) \times$ FINS $(\mathrm{mU} / \mathrm{l})] / 22.5$, of which the normal range of FINS was 3.0-24.9 U/ml and the normal value of HOMA-IR was 1. Moreover, Beckman Access DXI 800 Type analyzer 
Table III. Comparison of levels of inflammation-associated cytokines before and after intervention (mean \pm SD).

\begin{tabular}{lcc}
\hline & hs-CRP $(\mathrm{mg} / \mathrm{l})$ & TNF- $\alpha(\mathrm{ng} / \mathrm{l})$ \\
\hline Before intervention & $11.5 \pm 1.2$ & $138.1 \pm 11.4$ \\
After intervention & $6.1 \pm 0.1$ & $75.3 \pm 5.0$ \\
t value & 28.362 & 31.907 \\
P-value & $<0.001$ & $<0.001$ \\
\hline
\end{tabular}

Table IV. Comparison of ET-1 and NO levels before and after intervention (mean $\pm \mathrm{SD}$ ).

\begin{tabular}{lcc}
\hline & ET-1 (ng/l) & NO $(\mu \mathrm{mol} / 1)$ \\
\hline Before intervention & $98.6 \pm 6.21$ & $23.5 \pm 1.8$ \\
After intervention & $47.5 \pm 3.20$ & $28.9 \pm 2.1$ \\
t value & 46.262 & 12.348 \\
P-value & $<0.001$ & $<0.001$ \\
\hline
\end{tabular}

(Beckman Coulter Inc., Brea, CA, USA) as well as spectrophotometric method (Bio-Rad Laboratories, Inc., Hercules, CA, USA) were applied to measure the FINS. Detection of inflammatory cytokines: Double-antibody single-step sandwich method was adopted to detect the TNF- $\alpha$, of which the normal reference value in adults was 5-100 $\mathrm{ng} / \mathrm{l}$; latex particle-enhanced turbidimetric immunoassay was performed to measure hs-CRP, and the level $\leq 10 \mathrm{mg} / \mathrm{l}$ was regarded as the normal reference value in adults; radioimmunoassay was applied to examine ET-1 and the normal reference value in adults was 43.50-58.38 ng/l; enzyme-linked immunosorbent assay kit (Thermo Fisher Scientific, Inc., Waltham, MA, USA) was used for NO measurement.

Statistical analysis. Statistical Product and Service Solutions (SPSS) 21.0 (IBM Corp., Armonk, NY, USA) was applied for statistical processing; the measurement data were presented as mean \pm standard deviation and were calculated as percent $(\%)$. The levels of indexes related to blood lipid and HOMA-IR, ET-1, NO and inflammatory cytokines were compared by Student's t-test. ANOVA was used for comparison between multiple groups and the post hoc test was SNK test, and scatterplot was utilized for Spearman's correlation analysis. $\mathrm{P}<0.05$ indicated that the difference was statistically significant.

\section{Results}

Comparison of indexes related to blood lipid before and after intervention. Among the blood lipid indexes of the patients, the levels of TC, TG and LDL-C after intervention were significantly lower than those before intervention $(\mathrm{P}<0.05)$, while the HDL-C level after intervention was higher than that before intervention $(\mathrm{P}<0.05)$ (Table I).

Comparison of FINS and HOMA-IR levels before and after intervention. Compared with those before intervention, the

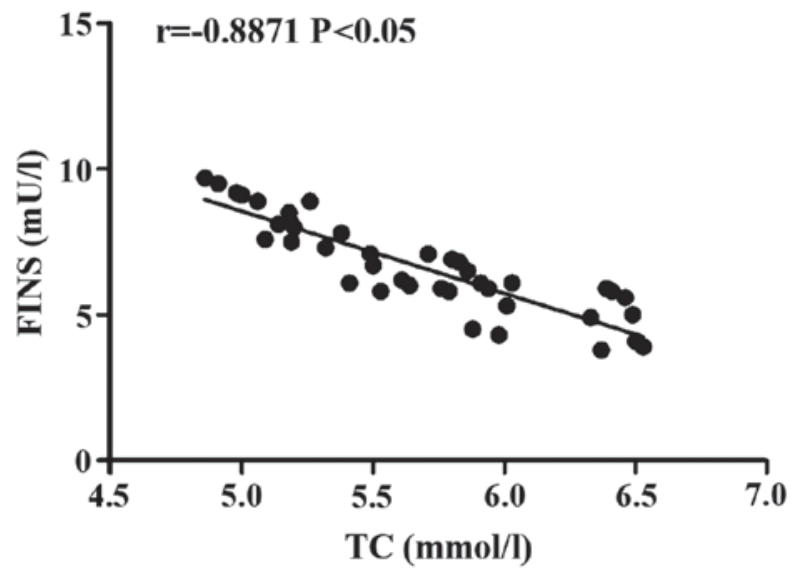

Figure 1. Correlation analysis of TC with FINS in the process of treatment on patients with diabetes mellitus and coronary heart disease using rosuvastatin. Correlation analysis of TC with FINS: The TC level was negatively correlated with FINS level $(\mathrm{r}=-0.8871, \mathrm{P}<0.05)$.

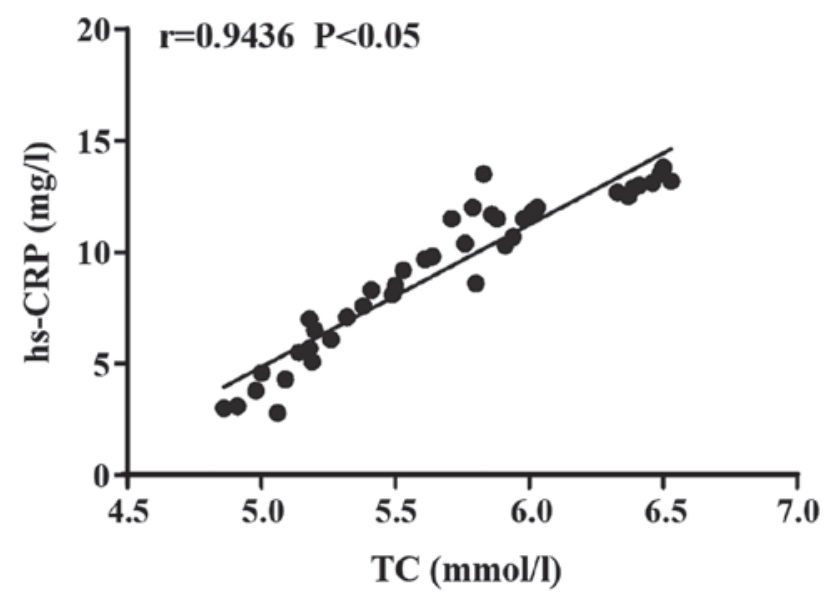

Figure 2. Correlation analysis of TC with hs-CRP in the process of treatment on patients with diabetes mellitus and coronary heart disease using rosuvastatin. Correlation analysis of TC with hs-CRP: The TC level was positively correlated with hs-CRP level $(\mathrm{r}=0.9436, \mathrm{P}<0.05)$.

level of FINS after intervention was remarkably elevated $(\mathrm{P}<0.05)$, while the HOMA-IR level after intervention was significantly reduced $(\mathrm{P}<0.05)$ (Table II).

Comparison of levels of inflammation-associated cytokines before and after intervention. After intervention, the levels of hs-CRP and TNF- $\alpha$ in the patients were obviously decreased compared with those before intervention $(\mathrm{P}<0.05)$ (Table III).

Comparison of ET-1 and NO levels before and after intervention. Compared with that before intervention, the ET-1 level was decreased $(\mathrm{P}<0.05)$, while the $\mathrm{NO}$ level was elevated after intervention $(\mathrm{P}<0.05)$ (Table IV).

Correlation analysis of TC with FINS in the process of treatment on patients with diabetes mellitus and coronary heart disease using rosuvastatin. The TC level was negatively correlated with FINS level $(r=-0.8871, \mathrm{P}<0.05)$ (Fig. 1). 


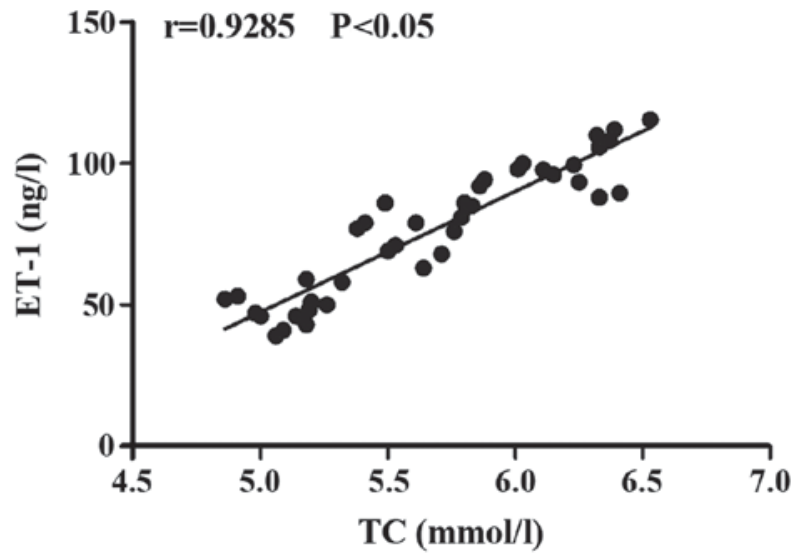

Figure 3. Correlation analysis of TC with ET-1 in the process of treatment on patients with diabetes mellitus and coronary heart disease using rosuvastatin. Correlation analysis of TC with ET-1: The TC level was positively correlated with ET-1 level $(\mathrm{r}=0.9285, \mathrm{P}<0.05)$.

Correlation analysis of TC with hs-CRP in the process of treatment on patients with diabetes mellitus and coronary heart disease using rosuvastatin. The TC level was positively correlated with hs-CRPlevel $(r=0.9436, p=0.000<0.05)$ (Fig. 2).

Correlation analysis of TC with ET-1 in the process of treatment on patients with diabetes mellitus and coronary heart disease using rosuvastatin. The TC level was positively correlated with ET-1 level ( $\mathrm{r}=0.9285, \mathrm{P}<0.05)$ (Fig. 3).

\section{Discussion}

With the progression in aging of population, improvement in living standards and changes in lifestyle and dietary patterns in China, the proportion of type 2 diabetes mellitus complicated with coronary heart disease has increased significantly (9). Both type 2 diabetes mellitus and coronary heart disease are the most common kinds of chronic and incurable internal diseases at present, which have high disability rates and fatality rates (10). Type 2 diabetes mellitus can cause metabolism disorders of carbohydrates and lipids which are energy substances, thus accelerating the occurrence and development of coronary atherosclerosis and leading to severely adverse prognosis (11). So far, some studies have confirmed that the metabolic disorder of blood lipid is an independent risk factor for the onset of the above-mentioned diseases (12); therefore, it is very important for patients with type 2 diabetes mellitus and coronary heart disease to effectively regulate their blood lipid level. Rosuvastatin, a statin that is most commonly used to regulate the metabolism of blood lipid in current clinical practices, is mainly extracted by fermentation of Aspergillus terreus, which can effectively suppress the synthesis of endogenous cholesterol, thus lowering the blood lipid level (13). As a result, the role of rosuvastatin in regulating the blood lipid has been proved clinically. The effect of rosuvastatin, however, on endothelial functions and inflammatory response factors in patients with type 2 diabetes mellitus and coronary heart disease needs to be further elucidated.

In this research, rosuvastatin was utilized for treatment; by comparing the indexes related to blood lipid of the patients before and after intervention, it was found that the levels of TC, TG and LDL-C after intervention were significantly lower than those before intervention, while the HDL-C level after intervention was higher than that before intervention. It suggested that treatment with rosuvastatin can effectively regulate the blood lipid level of patients with type 2 diabetes mellitus and coronary heart disease. In terms of testing indexes related to diabetes mellitus, it was shown that compared with that before intervention, the level of FINS after intervention was remarkably higher, while the HOMA-IR level after intervention was significantly lower. It indicated that applying rosuvastatin to type 2 diabetes mellitus complicated with coronary heart disease has a significant value in improving insulin function in the body and ameliorating insulin resistance. Furthermore, the variations in levels of inflammation-associated cytokines and vascular endothelial functions before and after intervention were studied, and the results revealed that the levels of hs-CRP and TNF- $\alpha$ in the patients after intervention were obviously decreased compared with those before intervention; compared with those before intervention, the ET-1 level was decreased, while the NO level was elevated after intervention. It suggested that for type 2 diabetes mellitus complicated with coronary heart disease, treatment using rosuvastatin can remarkably reduce inflammatory responses in the body and improve the vascular endothelial functions. In addition, the correlations of changes in TC with those in FINS, hs-CRP and ET-1 in the process of treating patients with diabetes mellitus and coronary heart disease using rosuvastatin were analyzed, and it was discovered that the TC level was negatively correlated with FINS level but positively correlated with the levels of hs-CRP and ET-1.

As the most common kind of water-soluble drug utilized to regulate lipid metabolism at present, rosuvastatin has relatively strong cell membrane penetration ability, so that it can exert its clinical efficacy by quickly entering the cytoplasm and nucleus (14). As a selective HMG-CoA reductase inhibitor (15), the drug can effectively restrain the synthesis of precursor of cholesterol in the body, elevate the level of LDL receptor on the liver cell surface and promote its absorption, thus lowering the levels of TG, TC and LDL-C and raising the HDL-C level (16). Moreover, rosuvastatin can inhibit the signaling pathways in the process of cell signal transduction and strengthen its binding to lymphocyte function-associated antigen 1 (LFA-1), thus controlling the binding rate of lymphocytes to intracellular adhesion molecule-1 (ICAM-1) and exerting the immunosuppressive effects (17); as a result, the levels of inflammation-associated cytokines in the body are decreased. Therefore, after treatment with rosuvastatin, the levels of hs-CRP and TNF- $\alpha$ in the body are decreased significantly (18). At the same time, rosuvastatin can lower the messenger RNA (mRNA) expression of preproendothelin-1 (PPET-1), the precursor of ET-1, in the body and then reduce the activity of ET-1 and its binding rate to receptors (19); furthermore, it can increase the expression level of endothelial nitric oxide synthase and reduce the negative regulation of oxidized LDL on mRNA of endothelial nitric oxide synthase, thus increasing the NO level in the body (20).

In conclusion, for patients with type 2 diabetes mellitus and coronary heart disease, treatment with rosuvastatin can effectively lower the levels of blood lipids and regulate insulin 
functions; moreover, potent decrease in blood lipid levels has great significance in improving the vascular endothelial functions and reducing inflammatory responses.

\section{Acknowledgements}

Not applicable.

\section{Funding}

No funding was received.

\section{Availability of data and materials}

The datasets used and/or analyzed during the current study are available from the corresponding author on reasonable request.

\section{Authors' contributions}

GM and SB were responsible for the observation and the analysis of the indexes. GM collected and analyzed the general data of the patients. Both authors read and approved the final manuscript.

\section{Ethics approval and consent to participate}

This study was approved by the Ethics Committee of Center Hospital of Zibo (Zibo, China) (no. ZBCH2015-03). The patients were advised, and signed the informed consent

\section{Patient consent for publication}

Not applicable.

\section{Competing interests}

The authors declare that they have no competing interests.

\section{References}

1. Foley TR, Singh GD, Kokkinidis DG, Choy HK, Pham T, Amsterdam EA, Rutledge JC, Waldo SW, Armstrong EJ and Laird JR: High-intensity statin therapy is associated with improved survival in patients with peripheral artery disease. J Am Heart Assoc 6: e005699, 2017.

2. Liang M, Yang S and Fu N: Efficacy of short-term moderate or high-dose rosuvastatin in preventing contrast-induced nephropathy: A meta-analysis of 15 randomized controlled trials. Medicine (Baltimore) 96: e7384, 2017.

3. Kwon O, Kang SJ, Kang SH, Lee PH, Yun SC, Ahn JM, Park DW, Lee SW, Kim YH, Lee CW, et al: Relationship between serum inflammatory marker levels and the dynamic changes in coronary plaque characteristics after statin therapy. Circ Cardiovasc Imaging 10: e005934, 2017.

4. Roy S, Weinstock JL, Ishino AS, Benites JF, Pop SR, Perez CD, Gumbs EA, Rosenbaum JA, Roccato MK, Shah H, et al: Association of cognitive impairment in patients on 3-hydroxy3-methyl-glutaryl-CoA reductase inhibitors. J Clin Med Res 9: 638-649, 2017.

5. Ovrakh T, Serik S and Kochubiei O: Impact of atorvastatin and rosuvastatin on residual on-clopidogrel treatment platelet reactivity in patients with ischemic heart disease and type 2 diabetes mellitus after acute coronary syndrome. Georgian Med News 4 : 7-14, 2017 (In Russian).
6. Jellinger PS, Handelsman Y, Rosenblit PD, Bloomgarden ZT, Fonseca VA, Garber AJ, Grunberger G, Guerin CK, Bell DSH, Mechanick JI, et al: American Association of Clinical Endocrinologists and American College of Endocrinology guidelines for management of dyslipidemia and prevention of cardiovascular disease. Endocr Pract 23 (Suppl 2): 1-87, 2017.

7. Zhang W, Yi L, Jin C, Xu S, Bi X, Zhao Y, Qiu F, Fu G and Min W: The impact of rosuvastatin on the density score of coronary artery calcification in coronary artery disease patients with type 2 diabetes mellitus: Rationale and design of RosCal Study. Clin Drug Investig 36: 1023-1029, 2016.

8. Ullah F, Afridi AK, Rahim F, ur Rahman S and Ashfaq M: Efficacy of $5 \mathrm{mg}$ and $10 \mathrm{mg}$ rosuvastatin in type-2 diabetes mellitus with hypercholesteroalemia. J Ayub Med Coll Abbottabad 27: 564-568, 2015.

9. Xiong X, Liu H, Hua L, Zhao H, Wang D and Li Y: The association of HDL-apoCIII with coronary heart disease and the effect of statin treatment on it. Lipids Health Dis 14: 127, 2015.

10. Gungoren F, Besli F, Caliskan S, Polat U, Basar C and Serdar OA: Statin therapy may not effect NLR and MPV levels in patients with hypercholesterolemia: A retrospective study. Angiology 67: 536-540, 2016.

11. Koshelskaia OA, Vinnitskaia IV, Konko TI, Kravchenko ES, Suslova TE and Karpov RS: Comparative randomized study of the effects of long-term therapy with rosuvastatin and combination of atorvastatin and ezetimibe on carbohydrate metabolism and adipokines levels in patients with coronary artery disease and diabetes mellitus. Kardiologiia 55: 67-74, 2015 (In Russian).

12. Terao Y, Satomi-Kobayashi S, Hirata K and Rikitake Y: Involvement of Rho-associated protein kinase (ROCK) and bone morphogenetic protein-binding endothelial cell precursorderived regulator (BMPER) in high glucose-increased alkaline phosphatase expression and activity in human coronary artery smooth muscle cells. Cardiovasc Diabetol 14: 104, 2015.

13. Suthar KS, Vanikar AV and Trivedi HL: Acute kidney injury and quadriparesis due to rosuvastatin induced rhabdomyolysis - a case report. J Clin Diagn Res 9: OD08-OD09, 2015.

14. Laires PA, Ejzykowicz F, Hsu TY, Ambegaonkar B and Davies G: Cost-effectiveness of adding ezetimibe to atorvastatin vs switching to rosuvastatin therapy in Portugal. J Med Econ 18: 565-572, 2015.

15. Laufs U, Gertz K, Dirnagl U, Böhm M, Nickenig G and Endres M: Rosuvastatin, a new HMG-CoA reductase inhibitor, upregulates endothelial nitric oxide synthase and protects from ischemic stroke in mice. Brain Res 942: 23-30, 2002.

16. Halcox JP, Tubach F, Lopez-Garcia E, De Backer G, Borghi C, Dallongeville J, Guallar E, Medina J, Perk J, Sazova O, et al: Low rates of both lipid-lowering therapy use and achievement of low-density lipoprotein cholesterol targets in individuals at high-risk for cardiovascular disease across Europe. PLoS One 10: e0115270, 2015.

17. Ball T and McCullough PA: Statins for the prevention of contrast-induced acute kidney injury. Nephron Clin Pract 127: 165-171, 2014.

18. Puri R, Nissen SE, Shao M, Ballantyne CM, Barter PJ, Chapman MJ, Erbel R, Libby P, Raichlen JS, Uno K, et al: Sex-related differences of coronary atherosclerosis regression following maximally intensive statin therapy: Insights from SATURN. JACC Cardiovasc Imaging 7: 1013-1022, 2014.

19. Liu YH, Liu Y, Duan CY, Tan N, Chen JY, Zhou YL, Li LW and He PC: statins for the prevention of contrast-induced nephropathy after coronary angiography/percutaneous interventions: A metaanalysis of randomized controlled trials. J Cardiovasc Pharmacol Ther 20: 181-192, 2015.

20. Stegman B, Puri R, Cho L, Shao M, Ballantyne CM, Barter PJ, Chapman MJ, Erbel R, Libby P, Raichlen JS, et al: High-intensity statin therapy alters the natural history of diabetic coronary atherosclerosis: Insights from SATURN. Diabetes Care 37: 3114-3120, 2014.

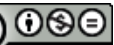

This work is licensed under a Creative Commons Attribution-NonCommercial-NoDerivatives 4.0 International (CC BY-NC-ND 4.0) License. 